\title{
Estudo de dois anos com animais reservatórios em área de ocorrência de leishmaniose tegumentar americana humana em bairro de urbanização antiga na cidade de Manaus-AM, Brasil
}

Jorge Augusto de Oliveira GUERRA ${ }^{1,3}$, Marcilene Gomes PAES ${ }^{1}$, Leíla Ines A.R. COELHO ${ }^{1,2,4}$, Marcus Luiz Barroso BARROS ${ }^{1,2}$, Nelson Ferreira FÉ ${ }^{1}$, Maria das Graças Vale BARBOSA ${ }^{1,3}$, Marcus Vinítius de Farias GUERRA ${ }^{1,2,3}$

\section{RESUMO}

Os autores descrevem o resultado de dois anos de investigação de um foco de Leishmaniose Tegumentar Americana (LTA), ocorrida em área urbanizada em um conjunto habitacional na cidade da Manaus-AM. Chama atenção o fato de que este não é o padrão de ocorrência de surtos da doença na região, e sim interrupção da transmissão após urbanização. Foram investigados os animais considerados reservatórios em potencial para a leishmaniose em domicílios humanos e em áreas de floresta adjacentes. Foram testados anticorpos contra Leishmania spp em amostras de sangue de cães e detectada reatividade pela reação de imunofluorescencia indireta em oito (20,51\%) dos examinados. Entre os animais silvestres examinados a espécie Didelphis marsupialis foi predominante, com 20 exemplares capturados, sendo encontrados homoflagelados em três destes e lesões suspeitas de leishmaniose cutanea em dois. Acredita-se que um assentamento populacional desordenado ocorrido nas adjacências tenha causado o deslocamento das populaçôes de vetores e reservatórios naturais em direção às casas do conjunto Hiléia propiciando o surto.

PALAVRAS-CHAVE

Reservatórios mamiferos, Leishmaniose Tegumentar, RIFI, Manaus.

\section{Two year study of reservoir animals in an old urban area with cutaneous Leishmaniasis occurrence in the city of Manaus-AM, Brazil}

\section{ABSTRACT}

This is the result of a two year follow-up of a Cutaneous Leishmaniasis (CL) foci in the Hileia urban neighborhood in Manaus. It is important to point out that this is not the usual pattern of Leishmaniasis occurrence in this area. The authors investigated the animal potential reservoir in households and in the surrounding forest area. Samples were tested for leishmaniasis antibodies by RIFI. Eight (20.51\%) of the examined domestic dogs showed reactivity. In captured wild animals, the Didelphis marsupialis was predominant in twenty specimens, three with homoflagelated in the blood and two with suspicious cutaneous lesions. We believe that the disordered population growth in the nearby area pushed the population of vectors and natural reservoir toward the homes in the Heleia neighborhood creating conditions for this outbreak.

\section{KEY-WORDS}

Reservoir, Cutaneous Leishmaniasis, Manaus, Brazil.

\footnotetext{
1 Fundação de Medicina Tropical de Medicina Tropical do Amazonas - FMTAM. Gerência de Leishmaniose. Avenida Pedro Teixeira, 25 - D. Pedro I. CEP 69040-000, Manaus - AM. Fax: (092) 238-3762. e-mail: jorge@fmt.am.gov.br

2 Universidade Federal do Amazonas - UFAM

3 Universidade do Estado do Amazonas - UEA

${ }^{4}$ Fundação Oswaldo Cruz. Centro de pesquisa Leonidas e Maria Deane
} 


\section{INTRODUÇÃO}

Em Manaus, no período de janeiro de 1985 a outubro de 1997, a ocorrência de casos de Leishmaniose Tegumentar Americana (LTA) alcançou 56\% do total ocorrido no estado do Amazonas. Nos últimos treze anos, a Fundação de Medicina Tropical do Amazonas (FMTAM) tem registrado em média de 1.000 casos anuais de LTA (Guerra et al. 1998). A doença ocorre principalmente na forma de Leishmaniose Cutânea (LC) (Romero et al. 2001), em surtos epidêmicos nos assentamentos populacionais recentes, próximos à floresta ou expostos diretamente em ambientes de mata primária (Guerra et al. 2003), passando posteriormente a apresentar-se como endemia de casos esporádicos (Barros et al. 1982; Paes, 1991).

Os mamíferos silvestres reconhecidos principalmente como reservatórios de LTA na região de Manaus são: Choloepus didactylus; Tamandua tetradactyla; Proechimys sp. e Didelphis marsupialis, sendo este último um dos hospedeiros mais importantes, pois tem o hábito de transitar no peridomicílio humano, estabelecendo um elo entre os ciclos de transmissão silvestre e peridomiciliar ( Arias \& Naiff, 1981; Lainson, 1983; Lainson et al. 1984; Guerra et al. 2006).

A transmissão da doença na região é atribuída aos flebótomos Lutzomyia umbratilis e Lutzomyia anduzei, considerados respectivamente vetores principal e secundário da Leishmania (Viannia) guyanensis, o parasito prevalente na etiologia da LTA/ LC, no município de Manaus (Arias \& Freitas, 1977; Paes, 1991).

O comportamento da doença no Bairro Planalto, onde se situa o conjunto Hiléia, assumia, nos últimos anos, comportamento de endemia com ocorrência de casos esporádicos pela proximidade das casas com a mata (Figura 1). Porém, em 1997, após 15 anos de implantação do conjunto, a ocorrência de 22 novos casos procedentes de determinadas ruas despertou interesse dos pesquisadores da FMTAM. Entre os casos observados, $64 \%$ residiam na rua 23 , que se limita com a mata em toda sua extensão (Paes et al., 1998).

Para se compreender a epidemiologia e a ocorrência desse novo surto, resolveu-se estudar os mecanismos envolvidos na reintrodução da transmissão da leishmaniose no conjunto Hiléia, Manaus (AM), assim como estimar a soroprevalência da infecção canina e o papel do cão como reservatório da LTA no bairro do Hiléia. Verificar a presença, na área, de mamíferos considerados reservatórios peri-domiciliares e silvestres de LTA, detectar a infecção por Leishmania e correlacionar os achados do inquérito sorológico com os animais e a ocorrência dos casos humanos e das espécies de flebotomíneos do gênero Lutzomyia transmissoras de Leishmaniose na área estudada.

\section{MATERIAL E MÉTODOS}

Foi estudada a ocorrência da LTA no Conjunto Hiléia, situado em um bairro de implantação antiga na zona Noroeste de Manaus, que faz limite com as matas do Aeroporto Internacional Eduardo Gomes (AIEG) (Figuras 1 e 2).

Foram proferidas inicialmente palestras educativas sobre Leishmaniose, visando esclarecer a comunidade a respeito dos objetivos do trabalho, assim como e orientar a adoção de medidas preventivas.

Para a avaliação dos cães foram selecionadas $25 \%$ das casas do conjunto e feitas entrevistas com os proprietários, exame desses animais quanto à presença de lesões cutâneas e coleta de amostra de sangue da orelha, em papel de filtro (eluato), para inquérito sorológico, feito pela Reação de Imunofluorescencia Indireta (RIFI) para a pesquisa de IgG, foi realizada segundo a descrição do kit de RIFI para diagnóstico da leishmaniose canina (Biomanguinhos-Rio de Janeiro/FIOCRUZ/MS/BR). Foram consideradas como amostras positivas, aquelas que apresentaram fluorescência em diluiçōes iguais ou maiores que 1/20. Quando constatadas lesões sugestivas de LTA, foram feitas biópsias em área de alopécia, pelagem ou úlceras, para exame direto, cultura e inoculaçôes em hamster (Mesocricetus auratus), objetivando o diagnóstico e o isolamento de cepas de Leishmania.

No inquérito dos animais silvestres foram utilizadas 26 armadilhas para captura de animais de pequeno, médio e grande

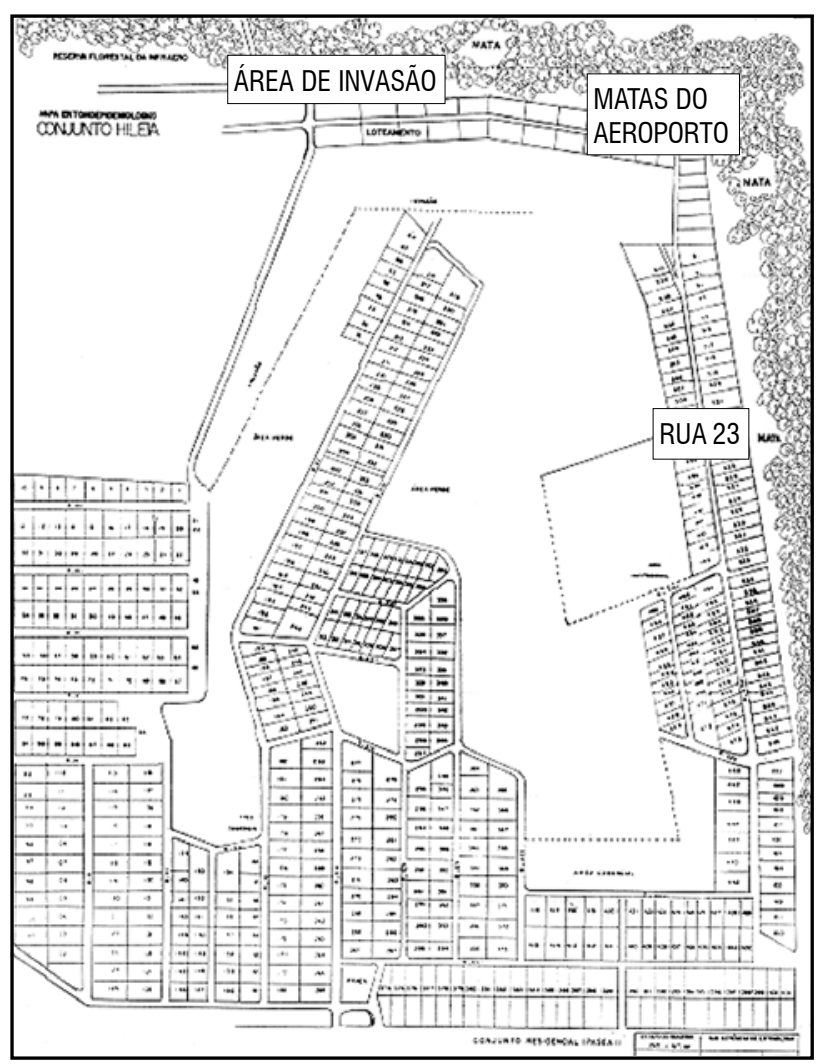

Figura 1 - Leishmaniose Tegumentar Americana em área urbanizada em Manaus, AM. Mapa da área estudada. 


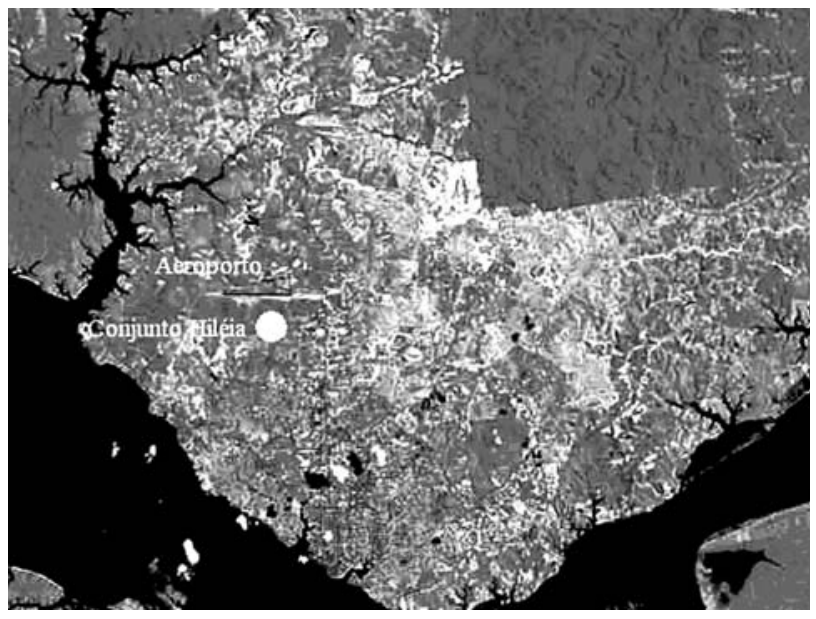

Figura 2 - Imagem Ladsat de Manaus, em destaque o Conjunto Hiléia local da área de estudo

porte, colocadas na mata da INFRAERO, a cerca de $500 \mathrm{~m}$ em relação às habitaçôes humanas. Alguns exemplares dos animais capturados foram sacrificados para exame histológico de vísceras e pele, e nos demais, foi retirado sangue venoso para realização de exames sorológicos, exames direto e a fresco. Fragmentos de vísceras foram macerados em solução salina inoculados em hamsters e semeados em meio bifásico NNN (Novy, MacNeal e Nicolle)/ Schneider's Insect Medium (SIGMA) contendo 10\% de soro fetal bovino. $\mathrm{O}$ material foi incubado a $28^{\circ} \mathrm{C}$, sendo realizados exames semanais a partir do quinto dia, durante seis semanas. Todos os animais capturados e não sacrificados foram marcados com uma argola de cobre em sua pata posterior e devolvidos a seu habitat natural.

Em estudos desenvolvidos paralelamente, foram realizados inquéritos entomológico e humano para avaliar a situação global da LTA na área.

\section{RESULTADOS}

Para o inquérito canino foram examinados 74 cães: 35 machos e 39 fêmeas. Entre esses, quinze apresentavam lesões; dois $(2,7 \%)$ apresentavam lesões não sugestivas de LTA e treze com lesōes traumáticas. Foi realizada sorologia (RIFI) de 39 cães $(52,7 \%)$, sendo oito $(20,51 \%)$ reativas (figura 3$)$.

Em relação aos animais silvestres, foram capturados 20 Didelphis marsupialis (mucura), um roedor Dasyprocta agouti (cutia) e três lacertídeos Tupinambis nigropuctatus (jacuraru) (Tabela 1). Em relação aos marsupiais, foram capturados onze machos e nove fêmeas, dos quais sete (35\%) apresentavam lesōes cutâneas, sendo duas sugestivas de LTA, porém, o exame direto destas lesões não foi constatado Leishmania. Foram sacrificados sete marsupiais (35\%) e realizados exames direto, histopatológico e cultura de pele, músculo esquelético, coração, fígado, baço, linfonodo, glândula anal, intestino, rim e cérebro. $\mathrm{Na}$ análise histológica não se encontrou parasito em nenhuma das peças. Além desses exames, nos hamsters foram realizadas inoculaçōes intradermicas (patas) e intraperitoneais com macerado de vísceras e sangue, sendo os resultados negativos para pesquisa de Leishmania. Nos marsupiais não sacrificados foram também realizados, de material coletado de lesōes e sangue venoso, pesquisa direta de Leishmania, cultura e inoculação em hamsters. No sangue venoso de dois animais foi observada a presença de hemoflagelados no exame direto e a fresco que, entretanto, não desenvolveram em cultura (Tabela 2). Em um outro animal, observou-se crescimento, em cultura, de hemoflagelados que aguardam caracterização. Do único roedor capturado (cutia), o qual não apresentava lesōes sugestivas de LTA, não foi possível coletar material para exames, pois este não sobreviveu em cativeiro.

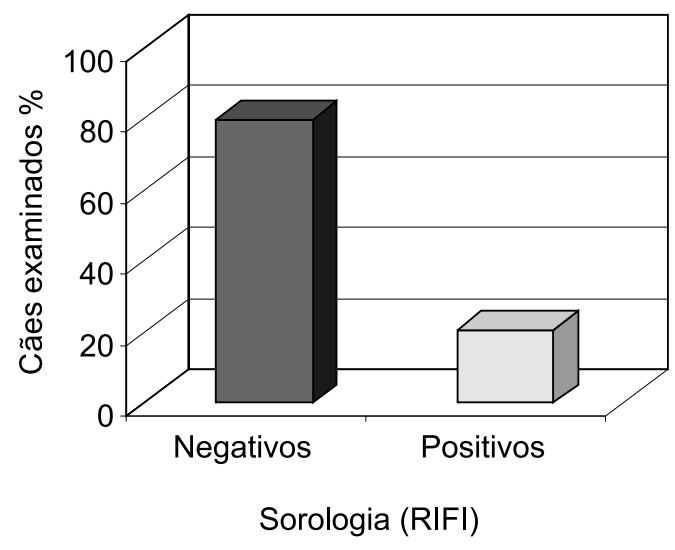

Figura 3 - Leishmaniose Tegumentar Americana em área urbanizada em Manaus, AM. Avaliação de 39 cães. Resultado da sorologia (RIFI).

Tabela 1 - Leishmaniose Tegumentar Americana em área urbanizada em Manaus. Avaliação dos reservatórios silvestres. Número de animais capturados e examinados

\begin{tabular}{cccc}
\hline Animais capturados & $\begin{array}{c}\mathrm{N}^{0} \text {. de animais } \\
\text { capturados }\end{array}$ & $\begin{array}{c}\mathrm{N}^{0} \text {. de animais } \\
\text { examinados }\end{array}$ & $\begin{array}{c}\mathrm{N}^{0} \text {. de animais } \\
\text { sacrificados }\end{array}$ \\
\hline Marsupiais & 20 & 20 & 07 \\
Roedor & 01 & - & - \\
Lacertídeos & 02 & - & - \\
TOTAL & 23 & 20 & 07 \\
\hline
\end{tabular}

Tabela 2 - Leishmaniose Tegumentar Americana em área urbanizada em Manaus. Exames laboratoriais realizados para estudo dos animais silvestres.

\begin{tabular}{ccc}
\hline Exames realizados & $\begin{array}{c}\text { Número de animais } \\
\text { examinados }\end{array}$ & $\begin{array}{c}\text { Exame direto positivo para } \\
\text { hemoflagelados }\end{array}$ \\
\hline Exame parasitológico direto & 20 & 02 \\
Exame histopatológico & 05 & - \\
Inoculação hamster & 08 & - \\
Cultura em meio NNN & 10 & 01 \\
\hline
\end{tabular}




\section{DISCUSSÃO}

Nos últimos anos diversos surtos de LTA têm sido registrados em Manaus. Guerra et al. (2001) registram 22 novos focos da doença de 1991 a 2000. As observações realizadas demonstram que, da mesma forma que neste estudo, as atividades de manejo florestal ou a manutenção de populações humanas em contato com áreas de mata primária propicia a ocorrência e manutenção de casos (Barret \& Senra, 1989; Andrade, 1998; Paes, 1991; Barros et al., 1998; Talhari et al. 1988), como parece ter ocorrido no conjunto do Hiléia.

$\mathrm{Na}$ região Amazônica, diversos estudos envolvendo isolamento e caracterizaçóes de cepas de Leishmania têm sido conduzidas (Grimaldi et al., 1989; Lainson, 1983; Lainson et al., 1994). Entretanto, nos lugares em que a LTA é causada pela L. (V.) guyanensis, reservatórios silvestres como o Didelphis marsupialis têm causado maior preocupação, uma vez que a adaptação em ambientes alterados os póe em circulação entre o meio florestal, onde se infectam, e o ambiente humano, onde é fonte de infecção a flebótomos (Andrade et al., 1998).

Arias \& Naiff (1981) e Arias et al. (1981) encontraram $D$. marsupialis infectados naturalmente por $L$. (V.) guyanensis em áreas periféricas de Manaus. No presente estudo, D. marsupialis foi o animal mais freqüente na área, demonstrando resultados compatíveis com o trabalho realizado por Andrade (1998) em um bairro da periferia de Manaus. Contudo, aguarda-se caracterização dos flagelados isolados de um desses animais por ocasião deste estudo.

Alteraçōes epidemiológicas levaram animais domésticos, como cães, a assumirem um papel importante na cadeia de transmissão da LTA em algumas áreas (Araújo Filho 1978; CampbellLendrum et al., 2001), chama atenção o fato de que reintrodução de transmissão não é o padrão de ocorrência de surtos da doença na região. Andrade (1998) observou, com muita freqüência, animais como gambás em foco semelhante em Manaus, onde também encontraram cães em ambiente peridomiciliar com sorologia positiva (RIFI) para leishmaniose, entretanto, naquele local, as pessoas e os cães adentravam uma reserva florestal. Guerra et al. (2002) realizaram inquérito sorológico canino, em áreas de transmissão e não transmissão humana de LTA em Manaus, os resultados obtidos a partir de soro obtido direto de punção venosa foram indicativos de infecção canina em áreas de transmissão humana de LTA.

Como não se encontrou parasitos por qualquer método utilizado e pela possibilidade de reaçôes cruzadas na sorologia canina, não se pôde atribuir aos cães o papel de reservatório. Entretanto, no estudo realizado paralelamente sobre os vetores no bairro do Hiléia, observou-se que a $L u$. umbratilis, principal transmissor da Leishmaniose em Manaus (Arias \& Freitas 1977; 1978; Paes, 1991), prevaleceu sobre as quatro espécies transmissoras mais importantes na região. Dessa forma, convém manter vigilância epidemiológica, principalmente em focos antigos em que a transmissão se mantém nos ambientes humanos próximos de floresta primária, pela possibilidade de os cães virem a ser um reservatórios da LTA, como tem sido demonstrado em outras áreas do país onde a transmissão é antiga e constante (Desjeux, 2001; Campbell-Lendrum et al., 2001; Madeira et al., 2003).

A captura de mucura ou gambá (D. marsupialis) sugere que este animal possa ter participado como um elo importante da transmissão da doença como reservatório secundário de $L$. (V.) guyanensis por apresentar uma boa capacidade de adaptação em ambientes perturbados, vivendo em transição entre a floresta e as residências (Andrade 1998; Guerra et al., 2006). Sua presença era referida com freqüência nos quintais dos moradores locais.

O manejo da mata próxima ao conjunto, determinado pelo processo de invasão populacional em 1997, possivelmente perturbou o ciclo silvestre e contribuiu com o microsurto fazendo com que os animais silvestres saíssem de seu habitat natural em busca de alimentos. Os vetores se adaptaram a essa condição, realizando hematofagia em animais domésticos e no homem, dando origem aos novos casos da doença. Acredita-se que as palestras educativas com orientações à comunidade possam ter contribuído para a interrupção dos casos. Até o momento, não se pôde atribuir aos cães o papel de reservatórios. Entretanto, novos estudos devem ser realizados em áreas semelhantes a fim de se estabelecer conclusões mais definidas, principalmente em relação ao papel dos vetores e reservatórios domésticos em áreas com semelhantes características.

\section{BIBLIOGRAFIA CITADA}

Andrade, S.L. 1998. Leishmaniose Tegumentar Americana em área de ocupação recente na periferia da cidade de Manaus, Estado do Amazonas, Brasil. Dissertação de Mestrado, Fundação Oswaldo Cruz, Rio de Janeiro, RJ. 206pp.

Araújo Filho, N.A. 1978. Epidemiologia da Leishmaniose Tegumentar Americana na Ilha Grande, Rio de Janeiro. Estudos sobre a infecção humana, reservatórios e transmissores. Dissertação de Mestrado. Universidade Federal do Rio de Janeiro, Rio de Janeiro, RJ. 148 pp.

Arias, J.R.; Freitas RA. 1977. On the vectors of cutaneus Leishmaniasis in Central Amazon of Brazil. Acta Amazonica, 7: 293-294,

Arias, J.R.; Freitas, R.A.; 1978. Sobre os vetores da leishmaniose cutanea na Amazônia Central do Brasill. 2: incidência de flagelados em fllebótomos selváticos. Acta Amazonica, 8(3): 387 196.

Arias, J.R.; Naif, R.D. 1981. The principal reservoir host of cutaneous leishmaniosis in the urban areas of Manaus, Central Amazon of Brazil. Memórias do Instituto Oswaldo Cruz, 76(3) :279-286.

Arias, J.R.; Naiff, R.D.; Miles, M.S.; Sousa, A.A. 1981. The opossum (Didelphis marsupialis(Marsupiala Didelphisae) as a reservoir host of Leishmania brasiliesis guyanensis in the Amazon basin of Brazil. Transactions of Royal Society of Tropical Medicine and Hygiene, 75: 537-541. 
Barret, T.V.; Senra; M.S. 1989. Leishmaniasis in Manaus, Brazil. Parasitology Today, 8: 255-257.

Barros, M.L.B.; Paes, M.G.; Talhari, S. 1982. Leishmaniose mucocutânea na Amazônia. Estudo dos casos diagnosticados em Manaus no período de 1976 a 1980. Anais Brasileiros de Dermatologia, 57: 152-54.

Barros, M.L.B.; Paes, M.G.; Toledo, L.M. 1998. Considerações sobre a produção da Leishmaniose Tegumentar Americana no Estado do Amazonas In: Espaço e Doença: um olhar sobre o Amazonas. Editora FIOCRUZ, Rio de Janeiro RJ. p. 105-113.

Brandão Filho, S.; Campbell-Lendrum, D.; Brito, M.E.F.; Shaw, J.J.; Davies, C.R. 1999. Epidemiological surveys confirm an incrasing burden of cutaneous leishmaniasis in north-east Brasil. Transactions of Royal Society of Tropical Medicine and Hygiene, 93: 488-494.

Campbell-Lendrum, D.; Dujardin, J.P.; Martinez, E.; Feliciangeli, M.D.; Perez, J.H.; Silans, L.N.M.P.; Desjeux, F. 2001. Domestic and peridomestic transmission of American Cutaneuous Leishmaniasis: Changing epidemiological patterns present new control opportunities. Memórias do Instituto Oswaldo Cruz, 96(2): 159-162.

Desjeux, P. 2001. The increase in risk factores for leishmaniasis worldwide. Transactions of Royal Society of Tropical Medicine and Hygiene, 95: 239-243.

Grimaldi, G.Jr. S.; Tesh, R.B. 1993. Leishmaniasis of the new world: Current concepts and implications for future research. Clinical and Microbiology Reviews, 6(3): 230-250.

Grimaldi, G. Jr.; Tesh, R.B.; McMahon-Pratt, D. 1989. A review of geographic distribution and epidemiology of leishmaniais in the New World. American Journal of Tropical Medicine and Hygiene, 41: 687-725.

Guerra, J.A.O.; Barros, M.L.B.; Guerra, M.V.F.; Talhari, S.; Paes, M.G. 1998. Leishmaniose Tegumentar no município de Manaus - Aspectos epidemiológicos. Revista da Sociedade Brasileira de Medicina Tropical, 31(supl. 1): 172.

Guerra, J.A.O.; Onety, A.C.; Santos, S.L.; Santos, F.G.C.; Talhari, S.; Paes, M.G. 2001. Situação da leishmaniose em Manaus na última década. Revista da Sociedade Brasileira de Medicina Tropical, 34(supl. 1): 244.

Guerra, J.A.O. Coelho, L.I.A.R.C.; Cabral, E.G.; Almeida, M.R.T.; Moura, M.A.; Paes, M.G. 2002. Comparação entre métodos para coleta de amostra de sangue em inquérito sorológico para leishmaniose em cães procedentes de áreas de transmissão e não transmissão humana de leishmaniose tegumentar americana no município de Manaus. NewsLab, 55: 72-80.
Guerra, J.A.O.; Talhari, S.; Paes, M.G.; Garrido, M.; Talhari, J.M. 2003. Aspectos clínicos e diagnósticos da leishmaniose tegumentar americana em militares simultaneamente expostos à infecção na Amazônia. Revista da Sociedade Brasileira de Medicina Tropical, 36(5): 587-590.

Guerra, J. A. O.; Ribeiro, J. A. S.; Coelho, L. I. A. R. C.; Barbosa, M. G. V.; Paes, G. P. 2006. Epidemiologia da Leishmaniose Tegumentar na comunidade São João, Manaus, Amazonas, Brasil. Cadernos de Saúde Publica 22: (11) 2319-27.

Lainson, R. 1983. The american leishmaniasis: some observations on their ecology and epidemiology. Transactions of Royal Society of Tropical Medicine and Hygiene, 77: 169-196.

Lainson, R.; Shaw, J.J.; Silveira, F.T.; Souza, A.A.A.; Braga, R.R.; Ishikawa, E.A.I. 1984. The dermal Leishmaniasis of Brazil with special reference to the eco-epidemiology of the disease in the Amazonian. Memórias do Instituto Oswaldo Cruz, 89(3): 435443.

Madeira M.F.; Uchôa C.M.A.; Leal, C.A.; Silva, R.M.M.; Duarte R.; Magalhães C.M.; Serra, C.M.B. 2003 Leishmania (Viannia) braziliensis em cães naturalmente infectados. Revista da Sociedade Brasileira de Medicina Tropical, 36(5): 551-555.

Paes, M.G.; Fé, N.F.; Coelho, L.I.A.R.C.; Oliveira, R.S.; Sousa, J.U.; Teixeira, M.R.; Romero, G.; Guerra, J.A.O. 1998. Sobre a ocorrência de leishmaniose tegumentar em rua de implantação antiga na cidade de Manaus (AM). Revista da Sociedade Brasileira de Medicina Tropical, 31(supl. 1): 75.

Paes, M.G. 1991. Estudo de quatro espécies de Lutzomyia França, 1924 (Diptera, Psychodidae) em área endêmica de Leishmaniose Tegumentar Americana na periferia de Manaus. Dissertação de Mestrado. Instituto Nacional de Pesquisas da Amazônia/ Fundação Universidade do Amazonas. Manaus, Amazonas. Páginas????

Romero, G.A.S.; Guerra, M.V.F.; Paes, M.G.; Macedo, V.O. 2001. Comparison of Cutaneous Leishmaniasis due to Leishmania (Viannia) braziliensis and L. (V.) guyanensis in Brazil: Clinical findings and diagnostic approach. Clinical of Infectous Diseases 32: 1304-1312.

Talhari, S.; Arias, J.A.; Cunha, M.G.S.; Naiff, R.B. Naiff, M.F.; Freitas, R.A.; Barrett, T. 1988. Leishmaniose no Estado do Amazonas Aspectos Epidemiológicos, Clínicos e Terapêuticos. Anais Brasileiros de Dermatologia, 63(6): 433-438.

WHO. 1990. Control ofleishmaniasis. Technical report series. Geneva, 793pp.

Recebido em 03/10/2003

Aceito em 20/02/2007 
